\title{
Spirulina supplementation: A double-blind, randomized, comparative study in young anemic Indian women
}

\author{
Lucia C. Leal-Esteban $^{\text {a }}$, Renata Campos Nogueira ${ }^{\text {a, " }}$, Mariana Veauvy ${ }^{\mathrm{a}}$, Benedict Mascarenhas ${ }^{\mathrm{b}}$, \\ Mandar Mhatre $^{\mathrm{c}}$, Sasikumar Menon ${ }^{\mathrm{c}}$, Bertrand Graz ${ }^{\mathrm{a}}$, Denis von der Weid ${ }^{\mathrm{a}}$

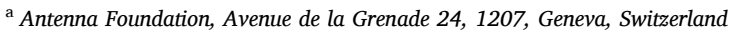 \\ ${ }^{\mathrm{b}}$ EnvisBE Solutions Pvt. Ltd., No.6, Yeshoda, Plot No. 179-A, LadyJamshedji Road, Dadar (W), Mumbai, 400028, India \\ ${ }^{\mathrm{c}}$ Ramnarain Ruia Autonomous College, Matunga, Mumbai, 400 019, India
}

\section{A R T I C L E I N F O}

\section{Keywords:}

Spirulina

Anemia

Clinical trial

Ferritin

Hemoglobin

Female

\begin{abstract}
A B S T R A C T
Background: The effect of spirulina (Arthrospira platensis), on haemoglobin and serum ferritine was tested in young women aged between 18 and 21 years and presenting an iron-deficiency anemia.

Methods: According to the allocation group, each participant daily consumed one placebo snack bar or one snack bar supplemented with $3 \mathrm{~g}$ of spirulina. At baseline, day 45, 60, and 90 after the first bar was consumed a complete blood count, serum ferritin, pregnancy test, and physical examination were performed.

Results: Anemia prevalence was found to be 33.7\% among the age range tested in Mumbai. In total, 181 women were screened, 61 of them were randomized and allocated into the two groups and finally 47 young women completed the study.

Conclusions: Spirulina or placebo snack bars were well tolerated. However, we did not observe significant changes in serum ferritin and hemoglobin in both groups at the end of the study.
\end{abstract}

\section{Introduction}

Anemia, most commonly diagnosed by a low hemoglobin level in the blood, is associated with increased morbidity and mortality. Although it affects one-third of the world's population the groups most vulnerable to anemia are children under 5 years old and women in reproductive age from low-income countries. ${ }^{1,2}$ Screening among non-pregnant women in the 15-45 years age group in northeastern states of India reported the incidence of anemia between 36 and 76\% depending on the region. In this population, anemia is in most cases caused by nutrition deficiencies. $^{3}$

Microalgae, like spirulina (Arthrospira platensis), contain highly nutritional molecules, such as proteins, amino acids, vitamins, and minerals. It can be easily incorporated in the regular diet providing a rich source of macro and micronutrients. Also, spirulina can be grown in diverse climatic conditions with higher productivity than traditional crops. ${ }^{4,5}$ Therefore, spirulina represents a suitable candidate to curb malnutrition in developing countries.
Clinical studies have provided scientific evidence supporting a positive correlation between nutritional supplementation with spirulina and improvement of nutritional parameters leading to reduced anemic condition. For instance, in young Indian girls (7-9 years old), the consumption of $1 \mathrm{~g}$ of spirulina in Panjiri five day a week during two months improved their hematological profile. ${ }^{6}$ Moreover, $1.5 \mathrm{~g}$ of spirulina supplementation in pregnant women (from 28 th week of amenorrhea to the 42nd day postnatal day) in Senegal showed higher gains in hemoglobin compared to the group allocated to iron and folic acid supplementation (IFAS). ${ }^{7}$ In children from Central Africa, the prevalence of severe anemia decreased from $20 \%$ to $6 \%$ after daily consumption of 10 $\mathrm{g}$ of spirulina during one month, ${ }^{8}$ HIV patients ${ }^{9,10}$ and seniors ${ }^{11}$ also showed an improvement in blood parameters after daily supplementation. So, affordable products that are appealing and have high nutritional content like spirulina might be a tool against malnutrition.

Based on the available data, we investigated if daily consumption of spirulina ( $3 \mathrm{~g}$ ) added into a snack bar known by the Indian population as "Chikki", would improve hemoglobin and serum ferritin levels in

\footnotetext{
* Corresponding author.

E-mail addresses: lealestebanlc@gmail.com (L.C. Leal-Esteban), rnogueira@antenna.ch (R.C. Nogueira), mveauvy@antenna.ch (M. Veauvy), ben_mas@

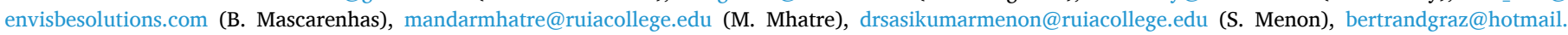
com (B. Graz), dweid@antenna.ch (D. von der Weid).
} 
anemic volunteers. 61 young mildly anemic women between 18 and 21 years old added in their diet a daily snack spirulina bar or snack placebo bar for 3 months according to the allocation group.

\section{Methods}

\subsection{Study design}

This study consisted in a randomized double blind clinical trial where the 61 selected participants were randomly allocated to consume placebo or spirulina snack bar and identified with alphabetic and numeric code for blinding. The chikki for assessment were randomized in a two-group parallel treatment design and identified with alphabet codes $(\mathrm{G} \& \mathrm{~N}$ ) for blinding. The randomization schedule was prepared using PROC PLAN in SAS v9.4 (SAS Institute Inc., Cary, NC, USA).

Chikki-Placebo and Chikki-Spirulina of equivalent weight were produced at Jay Chikki \& Snacks Pvt. Ltd., Mumbai, India as per formula developed for Antenna by EnvisBE Solutions Pvt. Ltd. The nutritional content and stability of placebo and spirulina snack bars were analyzed by Envirocare Labs \& Microchem Laboratory, Mumbai, India. The same laboratory analyzed the spirulina powder used in the production of Chikki-spirulina.
The participants performed 4 visits to the study site. During the first visit, they were screened for complete blood count, serum ferritin, pregnancy test, and a physical examination was performed. Selected participants were enrolled, allocated, and started the daily consumption of placebo or spirulina snack bar. The study field coordinators witnessed the consumption of snack bars daily. The daily consumption of snack bar was recorded in a subject diary by each subject. At day 45, 60, and 90 after the first bar was consumed, the participants visited the study site for assessment of complete blood count, serum ferritin, pregnancy test, and physical examination. Primary endpoints were hemoglobin and serum ferritin analysis at baseline, during, and after consumption.

According to the allocation group, each participant daily consumed one placebo snack bar or one spirulina snack bar containing $3 \mathrm{~g}$ of spirulina in the morning between $9.00 \mathrm{~h}$ and $12.00 \mathrm{~h}$ for 90 days. The participants continued with their normal lifestyle and followed all regular food habits during the study period. Both the participants and the field coordinators were blinded to group assignment.

From the 30 participants randomly allocated to the placebo group, 3 participants were lost to follow-up and 3 participants were excluded for noncompliance. From the 31 participants that were randomly allocated to the Spirulina group, 4 participants were lost to follow-up and 4 participants were excluded for noncompliance. Noncompliance included:

\section{$\Rightarrow \mathrm{CONSORT}$}

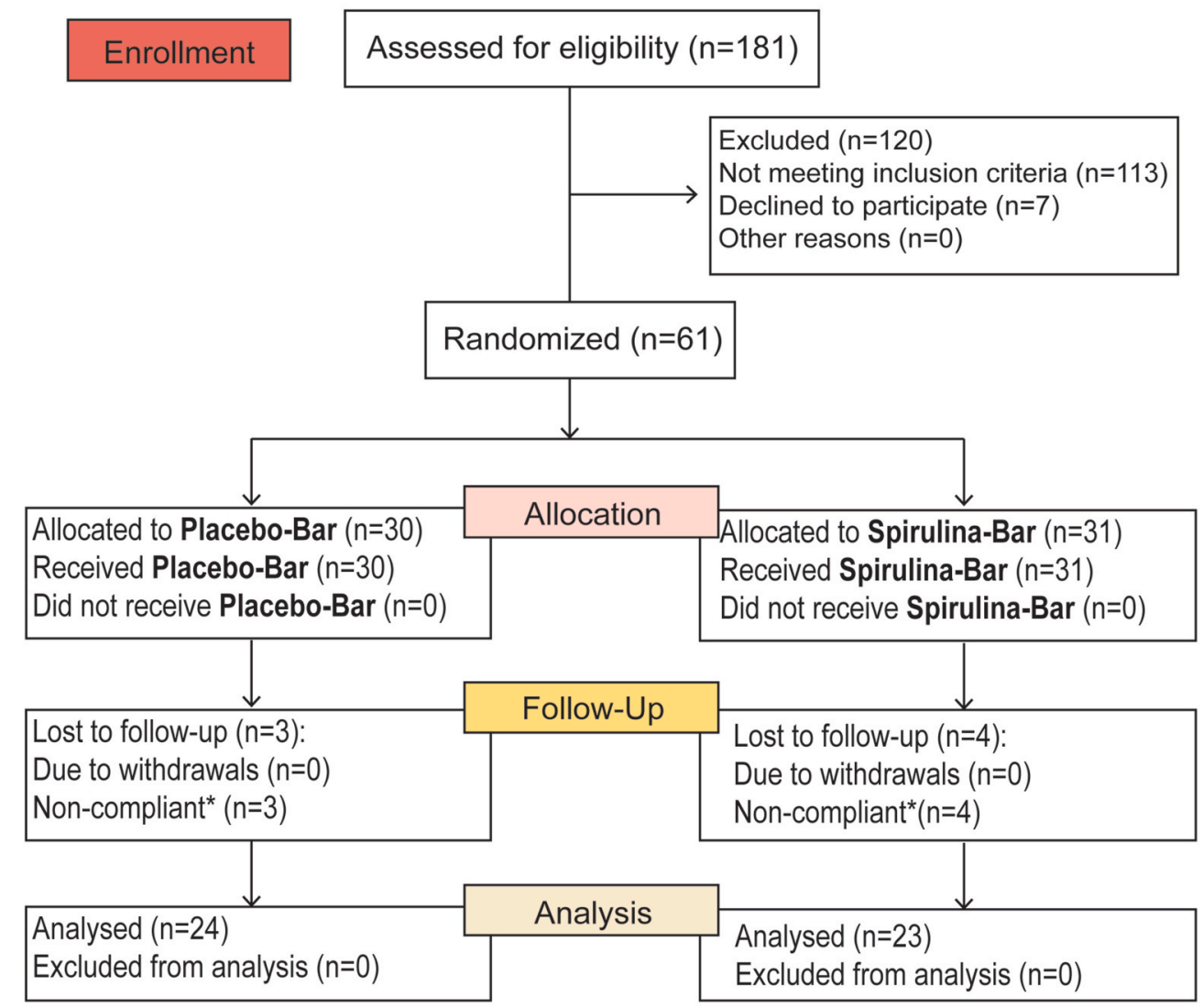

*Non-compliant: irregular consumption of the product, no clear recording of product consumption entries in the diary by subject, failed to reporting to study site on scheduled sample collection date.

Fig. 1. Study profile. Summary of the study design according to CONSORT guidelines. 
irregular consumption of the product, no clear recording of product consumption entries in the diary by subject, and failing to report to the study site on the scheduled sample collection date (Fig. 1).

\subsection{Sample}

The sample size calculation aimed to achieve the statistical significance for each group of data by a minimum number of thirty-three in phase one and twenty-eight in phase two. It was therefore planned to complete the study with fifty-four subjects in two phases.

\subsection{Study approval}

The study was conducted following CDSCO (Central Drugs Standard Control Organization), Ethical Guidelines for Biomedical Research on Human Participants, ICMR (Indian Council of Medical Research (2006) guidelines, ICH (Step 5) 'Guidance on Good Clinical Practice', Declaration of Helsinki (64th WMA General Assembly, Fortaleza, Brazil, October 2013) and with all applicable requirements of Principles of Good Laboratory Practices (OECD and Schedule L-1 of D\&C Rule 1945). The protocol was approved by the Institutional Ethics Committee of the Institute for advanced training and research in interdisciplinary science (Therapeutic Drug Monitoring Laboratory) under the number $\mathrm{CH}$ 170509-01.

\subsection{Participants}

Female volunteers, belonging to middle and lower middle class income group, between the ages of 18 and 21 (both inclusive) were recruited after field coordinators identified potential participants based on inclusion and exclusion criteria at the Therapeutic Drug Monitoring Laboratory in Mumbai, India. The recruitment process was by contact especially by word of mouth and by info passed between subjects. 181 volunteers were screened for complete blood count, serum ferritin, pregnancy test, and physical examination and were given informed consent. From the initial screening, 61 mildly anemic participants without serious clinical symptoms were enrolled in the study. The study was conducted during 2018 until 2019.

\subsection{Inclusion and exclusion criteria}

Inclusion criteria were stablished as: Females subjects, aged between 18 and 21 years old inclusive, with no serious clinical symptoms. The cut-off point for anemia at our study was taken as hemoglobin levels $\leq 120 \mathrm{~g} / \mathrm{L}$ as indicated by WHO for non-pregnant women above 15 years old. ${ }^{2}$ Selected participants had any hemoglobin levels $\leq 120 \mathrm{~g} / \mathrm{L}$ and any ferritin levels $\leq 15 \mu \mathrm{g} / \mathrm{L}$. Exclusion criteria included: illness or treatment for hypertension, high cholesterol, diabetes or thyroid dysfunction, allergies or sensitivity to food products, participation in another food, cosmetic or therapeutic trial or pregnancy and breastfeeding women.

\subsection{Statistical analysis}

Clinical data collection, analysis, and records, except the confidential and personal data of the subjects, were kept at EnvisBE Solutions Pvt. Ltd. (Mumbai, India). Two-way ANOVA followed by multiple $t$-test was used to evaluate the changes in all the variables over the study period. For statistical analysis, GraphPad Prism 8 was used and significance was accepted at $\mathrm{p}<0.05$.

\section{Results}

At the moment of enrollment in the study, the 24 participants from the placebo group had an average body mass index (BMI) of 20.13 (SD: 3.2 Min-Max: $15.2-27.4$ ) 33.3\% (8 out of 24) of them were following a vegetarian diet. The 23 participants from the spirulina group had an average 18.81 BMI (SD: 2.61 Min-Max: 15.7-28.8) and 26\% (6 out of 23) of them were following a vegetarian diet (Table 1). The difference in BMI between both groups were not statistically significant (p-value: 0.13).

Placebo and spirulina snack bars were analyzed for their nutritional content. Both bars showed similar values of most of the parameters measured, including energy, fat, carbohydrates, iron, and vitamin $C$. The parameters measured that showed the biggest differences between the two snack bars were protein and sodium content (Table 2). On the contrary, iron levels were very similar in both types of bars. The spirulina powder used in the production of the snack bars had $64.4 \mathrm{~g} / 100 \mathrm{~g}$ of protein and $34 \mathrm{mg} / 100 \mathrm{~g}$ of iron content.

At baseline, the placebo-snack bar group had an average hemoglobin level of $105.0 \mathrm{~g} / \mathrm{L}$ (SD: 11.0 Min-Max: 82.0-119.0) and the spirulina snack bar group had an average of $104.5 \mathrm{~g} / \mathrm{L}$ (SD:10.1 Min-Max: 86-118), p-value: 0.86 . After 45, 60, and 90 days of placebo or spirulina-snack bar consumption, the levels of hemoglobin in both groups did not significantly change (p-values: $0.79,0.46$ and 0.78 , respectively) (Fig. 2).

At baseline, the placebo-snack bar group had an average serum ferritin level of $3.55 \mu \mathrm{g} / \mathrm{L}$ (SD: 1.27 Min-Max: 1.30-5.8) and spirulinasnack bar group had an average of $4.54 \mu \mathrm{g} / \mathrm{L}$ (SD: 2.13 Min-Max: 2.00-9.40), p-value: 0.06 . After 45, 60, and 90 days of placebo or spirulina consumption, the levels of serum ferritin in both groups did not significantly change (p-values: $0.99,0.95$ and 0.28 , respectively) (Fig. 3).

All participants from both groups tolerated the snack bars well and did not show any adverse event during and/or after the completion of the study.

\section{Discussion}

The present study aimed to investigate if daily consumption of $3 \mathrm{~g}$ of spirulina added into a snack bar would ameliorate the anemia of childbearing age women.

We assessed 181 volunteers between 18 and 21 years old but most of them, 113 women, didn't meet the criteria because they were not anemic. In the region of Mumbai, we found that $33.7 \%$ (61) of the women tested were anemic and willing to participate in the program.

We applied low-cost technology to incorporate $3 \mathrm{~g}$ of spirulina into a generally accepted product (known as chikki bar), in an attempt to fill the nutritional gap in the Indian population. Both bars (placebo and spirulina) had similar nutritional content and were both well accepted by the participants.

There are clinical studies on nutritional supplementation with spirulina with anemic patients (mostly infants) but only a few evaluate hematological parameters. For instance, some of them report significant improvements in the ferritin as well as iron levels in Palestinian children younger than 5 years old after daily consumption of spirulina $(3 \mathrm{~g})$ for 3 months ${ }^{12}$ thus demonstrating the benefits of Spirulina in the treatment of child malnutrition. In line with this, some studies in Africa on the nutritional rehabilitation of malnourished and HIV-infected children. ${ }^{9,13}$ In the other hand, using a $5 \mathrm{~g}$ daily dosage during the same period ( 3 months), Branger et al. reported that spirulina did not bring any benefit compared to traditional nutrition in children aged from three months up to three years. However, the outcomes were not based

Table 1

Participants BMI and diet.

\begin{tabular}{lll}
\hline Parameter & Placebo-Snack bar & Spirulina-Snack bar \\
\hline BMI mean $\pm \mathrm{SD}\left(\mathrm{kg} / \mathrm{m}^{2}\right) *$ & $20.13 \pm 3.21$ & $18.81 \pm 2.61$ \\
Non-vegetarian diet $(\mathrm{n})$ & 16 out of 24 & 17 out of 23 \\
Vegetarian diet $(\mathrm{n})$ & 8 out of 24 & 6 out of 23 \\
\hline
\end{tabular}

* Means between placebo and spirulina are not significant. Unpaired $t$-test $(\mathrm{p}=$ 0.13 ). 
Table 2

Snack-bars nutritional content and microbial parameters.

\begin{tabular}{lll}
\hline Nutritional Parameter & Placebo-snack bar & Spirulina-snack bar \\
\hline Energy (kcal/100 g) & 386.5 & 381.7 \\
Fat $(\mathrm{g} / 100 \mathrm{~g})$ & 1.7 & 1.7 \\
Carbohydrates $(\mathrm{g} / 100 \mathrm{~g})$ & 87.8 & 82 \\
Protein $(\mathrm{g} / 100 \mathrm{~g})$ & 5 & 9.6 \\
Sodium $(\mathrm{mg} / 100 \mathrm{~g})$ & 254 & 475 \\
Iron $(\mathrm{mg} / 100 \mathrm{~g})$ & 241 & 243 \\
Vitamin C (mg/100 g) & 12.1 & 13.1 \\
Microbial Parameter & & \\
Total plate count $(\mathrm{cfu} / \mathrm{g})$ & $4.3 \times 10^{2}$ & $3.5 \times 10^{3}$ \\
Coliform count $(\mathrm{cfu} / \mathrm{g})$ & $<10$ & $<10$ \\
E. coli (per 25 g) & Absent & Absent \\
Staphylococcus aureus (per $25 \mathrm{~g})$ & Absent & Absent \\
Salmonella (per 25 g) & Absent & Absent \\
Yeast and Mold count (cfu/g) & $<10$ & $<10$ \\
\hline
\end{tabular}

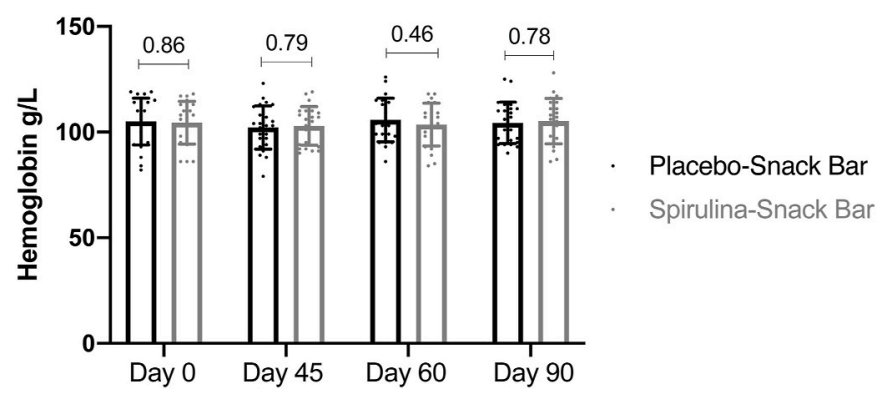

Fig. 2. Hemoglobin levels. Bars show the mean and standard deviation of hemoglobin levels for each study group at four different time points. P-values obtained from the statistical analysis between the two study groups are shown.

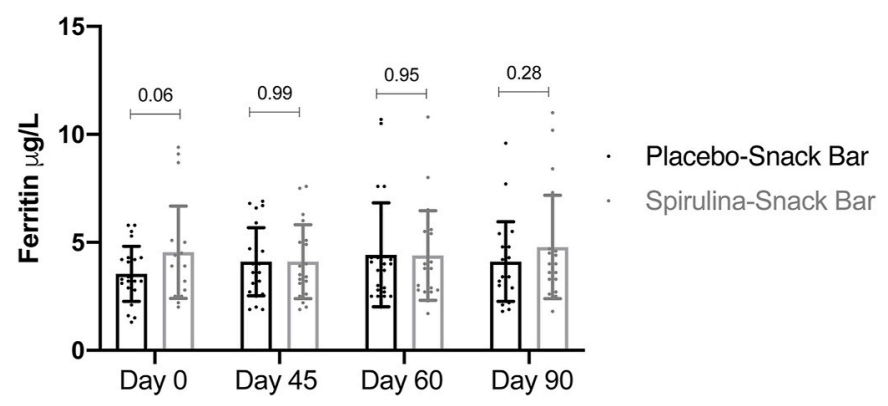

Fig. 3. Serum ferritin levels. Bars show the mean and standard deviation of serum ferritin levels for each study group at four different time points. P-values obtained from the statistical analysis between the two study groups are shown.

on biochemical measurements but height per age, weight for height group evolution, and the corresponding Z-score at 60 and 90 days instead. ${ }^{14}$

To our best knowledge, there are two clinical studies using spirulina supplementation on anemic women comprising the age range 18-21 years old. Madhusnata and colleagues reported that after one year of daily supplementation with $300 \mathrm{mg}$ of spirulina, 257 anemic women from ages 36-76 years considerably increased their hemoglobin levels. ${ }^{3}$ Mani and colleagues evaluated the daily supplementation with $5 \mathrm{~g}$ of Spirulina powder (as syrup or parathas) in the diet of young anaemic girls, 18-22 years, for 30 days and observed a mean increase of $10.33 \%$ in the blood hemoglobin levels. ${ }^{15}$

Although our daily dosage $(3 \mathrm{~g})$ is comprised in the above-mentioned studies, we did not observe an increase in blood hemoglobin or serum ferritin levels. This may be explained by several reasons. First, the duration of the study; 3 months of daily consumption of $3 \mathrm{~g}$ of spirulina in snack bars may be a short period and perhaps we would have to extend the length of the study, as done by Madhusnata and colleagues, to observe significant changes. Second, it was recently reported that Chikki snack bars contains oxalates and phytates that can inhibit iron absorption, thus affecting the uptake of nutrients present in spirulina. One can consider that the iron contained in the $3 \mathrm{~g}$ of spirulina supplemented in the snack bars were not sufficient to overcome the effect of oxalates and phytates. ${ }^{16}$ Besides, the iron content was very similar in the chikki bars, with and without spirulina (Table 2) and the spirulina powder had an amount of iron that is perhaps inferior from the amount used in other trials. Although some studies observed that the relation between iron supplementation and decreasing anemia may not be straightforward, as iron supplementation in itself may not be able to overcome other nutritional deficiencies because other nutrients seem to play an important role, ${ }^{17}$ of which some might have been in insufficient supply in both groups. Additionally, as the main study limitation, the lack of deworming might also explain the results found in our study as anemia is strongly associated with moderate and heavy hookworm infection. ${ }^{18}$ Although in an urban community in India it was found that under-nutrition and low per capita monthly income had a statistically significant relationship to anaemia which were not the case of intestinal parasitic and malaria infection ${ }^{19}$ this question remains open. Because of cultural issues, we decided not to perform deworming before their inclusion in the trial. Among women in this community, collecting stool for analysis is perceived as shameful and they threatened to leave the study if that was required. Furthermore, as it is not allowed to provide deworming medication without the stool analysis in a clinical study, we decided to proceed with the protocol without deworming the participants.

Our results highlight the importance of hemoglobin and ferritin screening in young female Indian populations and the acceptability of nutritional supplementation in such populations. These results bring new information to practitioners that aim to treat the young female patients without any severe symptoms. Future interventionist studies might consider the population perceptions about stool analysis to exclude the possible effect of parasitic infection on anemia.

\section{Conclusion}

The supplementation with $3 \mathrm{~g}$ of spirulina into a snack bar (known as chikki) was well tolerated and well accepted in young anemic women between 18 and 21 years but did not significantly change the levels of serum ferritin and hemoglobin after 3 months of daily consumption.

\section{Funding}

This research was funded by Antenna Foundation, Geneva, Switzerland.

\section{Declaration of competing interest}

No potential conflict of interest was reported by the authors.

\section{Acknowledgments}

We express our gratitude to all the women that participated in this study.

\section{References}

1 Kassebaum NJ, Jasrasaria R, Naghavi M, et al. A systematic analysis of global anemia burden from 1990 to 2010. Blood. 2014;123(5):615-624.

2 Chaparro CM, Suchdev PS. Anemia epidemiology, pathophysiology, and etiology in low- and middle-income countries. Ann N Y Acad Sci. 2019;1450(1):15-31.

3 De M, Halder A, Chakraborty T, et al. Incidence of anemia and effect of nutritional supplementation on women in rural and tribal populations of eastern and northeastern India. Hematology. 2011;16(3):190-192.

4 Christaki E, Florou-Paneri P, Bonos E. Microalgae: a novel ingredient in nutrition. Int J Food Sci Nutr. 2011;62(8):794-799. 
5 Matos J, Cardoso C, Bandarra NM, Afonso C. Microalgae as healthy ingredients for functional food: a review. Food Funct. 2017;8(8):2672-2685.

6 Sachdeva R, Kaur R, Sangha JK. Effect of supplementation of spirulina on the haematological profile and intellectual status of school girls (7-9 years). J Hum Ecol. 2004;15(2):105-108.

7 Niang K, Ndiaye P, Faye A, et al. Spirulina supplementation in pregnant women in the Dakar region (Senegal). Open J Obstet Gynecol. 2017;7:147-154.

8 Matondo FK, Takaisi K, Nkuadiolandu AB, Kazadi Lukusa A, Aloni MN. Spirulina supplements improved the nutritional status of undernourished children quickly and significantly: experience from kisantu, the Democratic Republic of the Congo. Int $J$ Pediatr. 2016;2016:1296414.

9 Azabji-Kenfack M, Dikosso SE, Loni EG, et al. Potential of spirulina platensis as a nutritional supplement in malnourished HIV-infected adults in sub-saharan Africa: Randomised, single-blind study. Nutr Metab Insights. 2011;4:29-37.

10 Ngo-Matip ME, Pieme CA, Azabji-Kenfack M, et al. Impact of daily supplementation of Spirulina platensis on the immune system of naïve HIV-1 patients in Cameroon: a 12-months single blind, randomized, multicenter trial. Nutr J. 2015;14:70.

11 Selmi C, Leung PS, Fischer L, et al. The effects of Spirulina on anemia and immune function in senior citizens. Cell Mol Immunol. 2011;8(3):248-254.
12 Abed E, Thab A, Suliman E, Mahmoud A. Impact of spirulina on nutritional status, haematological profile and anaemia status in malnourished children in the Gaza Strip: randomized clinical trial. Matern Pediatr Nutr. 2016;110:2.

13 Masuda K, Inoue Y, Inoue R, et al. Spirulina Effectiveness Study on Child Malnutrition in Zambia. 2014.

14 Branger B, Cadudal JL, Delobel M, et al. [Spiruline as a food supplement in case of infant malnutrition in Burkina-Faso]. Arch Pediatr. 2003;10(5):424-431.

15 Uliyar M, Alefia S, Uma I, Panam P. The effect of Spirulina supplementation on blood haemoglobin levels of anaemic adult girls. J Food Sci Technol. 2000;37(6):642-644.

16 Batra V, Lomash H, Ganguli A. Fermentation of groundnut brittle by Lactococcus lactis produces $\gamma$-amino butyric acid and enhances nutritional quality and safety. Food Quality and Safety. 2018;2(2):83-87.

17 Swaminathan S, Ghosh S, Varghese JS, Sachdev HS, Kurpad AV, Thomas T. Dietary iron intake and anemia are weakly associated, limiting effective iron fortification strategies in India. J Nutr. 2019;149(5):831-839.

18 Smith JL, Brooker S. Impact of hookworm infection and deworming on anaemia in non-pregnant populations: a systematic review. Trop Med Int Health. 2010;15(7): 776-795.

19 Khan MM, Rao GK, Viqarunnisa. Relationship of intestinal parasitism, malaria and under-nutrition to prevalence of anaemia in an urban community. J Commun Disord. 1990;22(2):116-119. 